\title{
Effects of Ultrasonic Irradiation on Preparation of Titanium Dioxide Photocatalyst by Anodic Oxidation Method
}

\author{
Yosuke Komai $^{1, *}$, Yoshiteru Mizukoshi ${ }^{2}$, Kenji Okitsu ${ }^{1}$, \\ Rokuro Nishimura ${ }^{1}$ and Naoya Masahashi ${ }^{2}$ \\ ${ }^{1}$ Department of Materials Science, Graduate School of Engineering, Osaka Prefecture University, Sakai 599-8531, Japan \\ ${ }^{2}$ Institute for Materials Research, Tohoku University, Sendai 980-8577, Japan
}

Titanium dioxide $\left(\mathrm{TiO}_{2}\right)$ photocatalyst was fabricated by anodic oxidation on pure titanium in an electrolyte of a sulfuric acid, and effect of ultrasonic irradiation during anodic oxidation was explored. It was found that ultrasonic irradiation increased crystallinity of the anodic oxide. The hydrophilicity of the as-anodized oxide was improved by applying ultrasonic irradiation, and it is similar to that of the annealed oxide without applying ultrasonic irradiation. In contrast, the photoactivity of the as-anodized oxide was not altered by applying ultrasonic irradiation. [doi:10.2320/matertrans.MAW200912]

(Received April 24, 2009; Accepted July 3, 2009; Published August 25, 2009)

Keywords: photocatalysis, anodic oxidation, titanium oxide, ultrasonic irradiation

\section{Introduction}

Titanium dioxide $\left(\mathrm{TiO}_{2}\right)$ has attracted considerable interests due to its photocatalytic activity and hydrophilicity, and extensive studies have been carried out to elucidate the mechanism of the characteristics and expand its practical applications. ${ }^{1)}$

Ultrasound has been applied to fabricate novel materials for more than decades. ${ }^{2,3)}$ Chemical effects of ultrasound are originated from cavitation phenomena, namely adiabatical collapse of cavitation bubbles, which are generated in the medium under high power ultrasonic irradiation. ${ }^{4}$ Through the collapse of bubbles, generation of hot reaction sites ${ }^{5)}$ accompanied by formation of radicals such as hydroxyl radicals ${ }^{6)}$ has been proposed. Further, shock wave, ${ }^{7)}$ and jet streams on the heterogeneous surface ${ }^{8,9)}$ have been reported as other effects. Sonochemical effects on electrochemical reaction have been widely explored, because ultrasound provides degassing, promotion of mass transfer, and destruction of electrical double layer. ${ }^{10)}$ However, sonochemical effects on anodic oxide during anodic oxidation have not been investigated except a few reports. ${ }^{11,12)}$

The authors studied an anodic oxidation to form photocatalyst $\mathrm{TiO}_{2}$ on titanium and its alloys, ${ }^{13-16)}$ because it provides adhesive strength at the interface with a low interfacial strain due to a small difference in the thermal expansion coefficient between titanium and oxide. In this study, we have investigated the photocatalytic characteristics of the oxide prepared by anodic oxidation in an electrolyte of a sulfuric acid expecting an enhancement of oxide formation by applying ultrasonic irradiation.

\section{Experimental}

Pure titanium plates (cp-Ti, grade I) with dimensions of $1.0 \times 2.0 \times 0.1 \mathrm{~cm}^{3}$ were prepared for use as the anode in anodic oxidation. A constant current of $50 \mathrm{~mA} / \mathrm{cm}^{2}$ was applied to the Ti plate in a sulfuric acid electrolyte of 0.02

*Graduate Student, Osaka Prefecture University

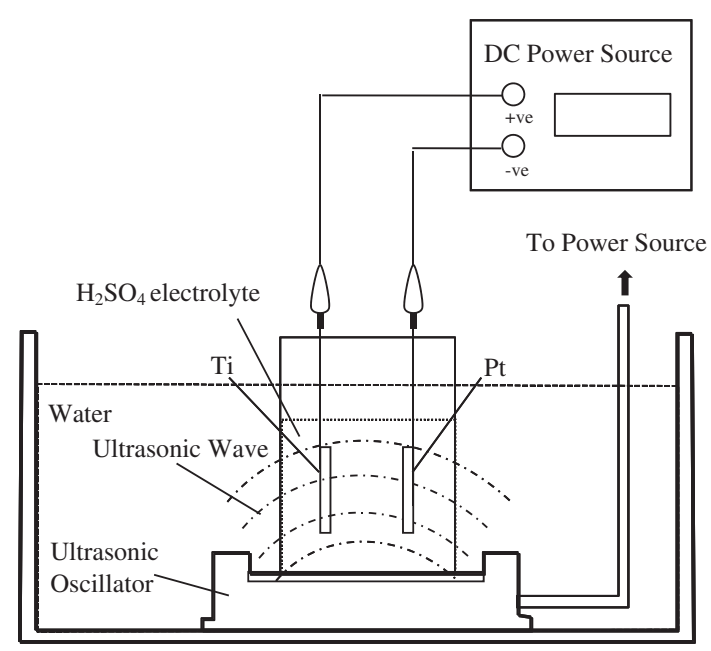

Fig. 1 Experimental setup for anodic oxidation with ultrasonic irradiation.

and $0.79 \mathrm{M}$, and a Pt mesh electrode was used as the cathode. The anodic oxidation was controlled galvanostatically with applying the conversion voltage ranging from 40 to $210 \mathrm{~V}$, and the time duration of oxidation was fixed at $0.5 \mathrm{~h}$. The anodized electrode was rinsed with distilled water and dried at room temperature, followed by annealing at $723 \mathrm{~K}$ for $5 \mathrm{~h}$ in air. The electrolyte was irradiated with ultrasound of $6 \mathrm{~W} / \mathrm{cm}^{2}$, and $200 \mathrm{kHz}$ (TA-4021, KAIJO). Figure 1 shows the experimental setup for anodic oxidation with ultrasonic irradiation.

The crystallographic structure of the anodized oxide was determined by X-ray diffraction (XRD, PANalytical X'Pert diffractometer, Phillips). Microstructure observations were conducted with a scanning electron microscopy (SEM, VE8800 , Keyence) at an operating voltage of $20 \mathrm{kV}$.

The photocatalytic activity under UV light illumination with an intensity of $1.5 \mathrm{~mW} / \mathrm{cm}^{2}$ at the surface of the reaction cell was investigated using methylene blue (MB) bleaching tests. The MB bleaching test was conducted by measuring the absorbance of $\mathrm{MB}$ at $664 \mathrm{~nm}$ using a UV-vis spectrophotometer (V-550DS, Jasco) after UV illumination and the 


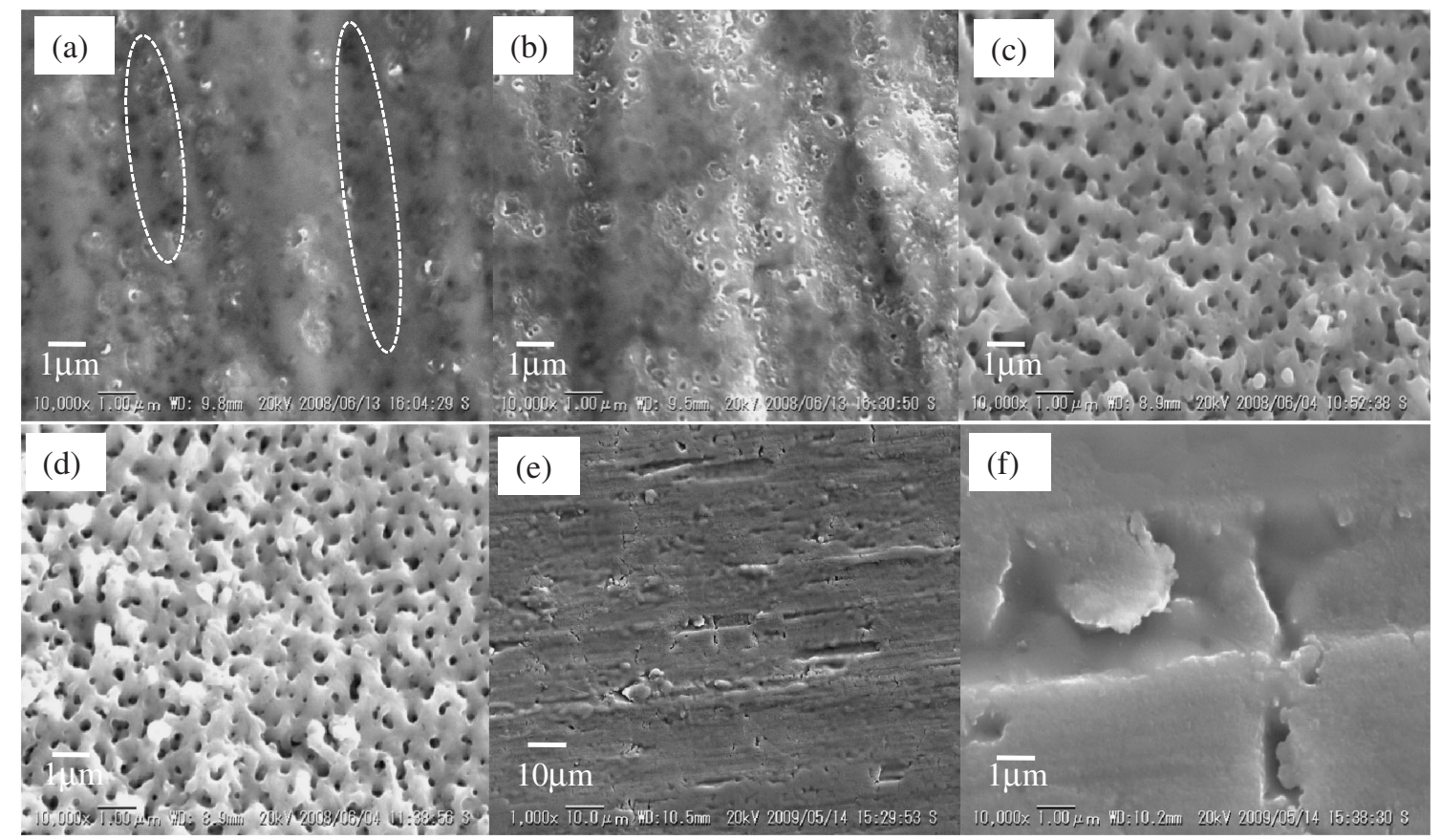

Fig. 2 SEM images of the oxides anodized in an electrolyte with sulfuric acid concentrations of $0.02 \mathrm{M}$ (a), (b), $0.79 \mathrm{M}$ (c), (d) with ultrasonic irradiation (b), (d), without ultrasonic irradiation (a), (c) and the titanium substrate (e), (f).

detail was ascribed in elsewhere. ${ }^{13-15)}$ Degradation rate of MB was calculated according to the following equation, $\mathrm{D}_{\mathrm{MB}}=\left\{\left(\mathrm{A}_{\text {blank }}-\mathrm{A}_{\text {measured }}\right) / \mathrm{A}_{\text {blank }} \times 100\right\}$, wherein $\mathrm{A}_{\text {blank }}$ and $A_{\text {measured }}$ are absorbance at $664 \mathrm{~nm}$ without and with $\mathrm{TiO}_{2}$ photocatalyst, respectively. In order to evaluate hydrophilicity of the anodic oxides, the contact angle of distilled water $(0.1 \mathrm{~mL})$ was measured by using a goniometer system (Drop Master 500, Kyowa Interface Science), and the contact angle was measured every half hour during UV illumination with an intensity of $0.2 \mathrm{~mW} / \mathrm{cm}^{2}$ at the surface of the sample.

\section{Results and Discussion}

\subsection{Microstructures}

Figure 2 shows the microstructures of the oxides anodized in an electrolyte of sulfuric acid concentrations of 0.02 (a), (b) and $0.79 \mathrm{M}$ (c), (d) with ultrasonic irradiation (b), (d) and without ultrasonic irradiation (a), (c). When the sulfuric acid concentration is $0.02 \mathrm{M}$, the thickness of the anodized oxide is thin. This is supported by charge-up distribution observation in SEM images, where the substrate is observed as charge-up free as circled in (a). As reference, the microstructures of the titanium substrate are shown in (e), (f). With ultrasonic irradiation during anodic oxidation, glassy anodized oxide with submicron sized pores is observed as shown in (b). The observed pores are distributed inhomogeneously in shape and size, and their frequency is quite low. With an increase in the concentration of sulfuric acid of $0.79 \mathrm{M}$, round-shaped pores appear homogeneously in the oxide as shown in (c). However, ultrasonic irradiation did not alter the microstructure of the anodic oxides as shown in (d). These results imply that the effect of ultrasonic irradiation on microstructure evolution is recognized when the sulfuric acid concentration in the electrolyte is low. Detail will be discussed in the next section.

\subsection{Crystal structure}

Figure 3 shows the XRD profiles of the oxides anodized in an electrolyte with sulfuric acid concentrations of $0.02 \mathrm{M}$ (a) and $0.79 \mathrm{M}$ (b). The relative peak intensity ratio of the predominant oxide peak to hcp Ti $(101)\left(2 \theta=40.1^{\circ}\right)$ increases with an increase in the sulfuric acid concentration and the conversion voltage, indicating that the thickness of the oxide layer increases with the sulfuric acid concentration and the conversion voltage. When the sulfuric acid concentration is $0.02 \mathrm{M}$, the intensity of anatase (101) $\left(2 \theta=25.4^{\circ}\right)$ increases with the conversion voltage and ultrasonic irradiation as compared to that without ultrasonic irradiation as shown in (a). In contrast, similar variation is not recognized when the sulfuric acid concentration is $0.79 \mathrm{M}$. The predominant oxide phase converts from anatase to rutile when the sulfuric acid concentration is $0.79 \mathrm{M}$ and the conversion voltage is $160 \mathrm{~V}$ as shown in (b). In order to evaluate the crystallinity of the oxides, the full width at half maximum (FWHM) of anatase (101) was calculated and the result is shown in Fig. 4. When the sulfuric acid concentration is $0.02 \mathrm{M}$ (a), the FWHM of anatase (101) of the as-anodized oxides decreases with the conversion voltage and ultrasonic irradiation, whereas that of the annealed oxides is essentially constant irrespective of the conversion voltage and ultrasonic irradiation. These results suggest that the crystallinity of anatase phase is improved by ultrasonic irradiation and an application of ultrasonic irradiation has the same effect on improving crystallinity with annealing. It is found that the as-anodized oxides with ultrasonic irradiation exhibit low FWHM as compared to that without ultrasonic irradiation, suggesting that the crystallinity increases with ultrasonic irradiation. On the other hand, similar result is not recognized when the sulfuric acid concentration is $0.79 \mathrm{M}(\mathrm{b})$, which is the same tendency with the result of microstructure evolution in the anodic oxides. This is quite interesting in considering 

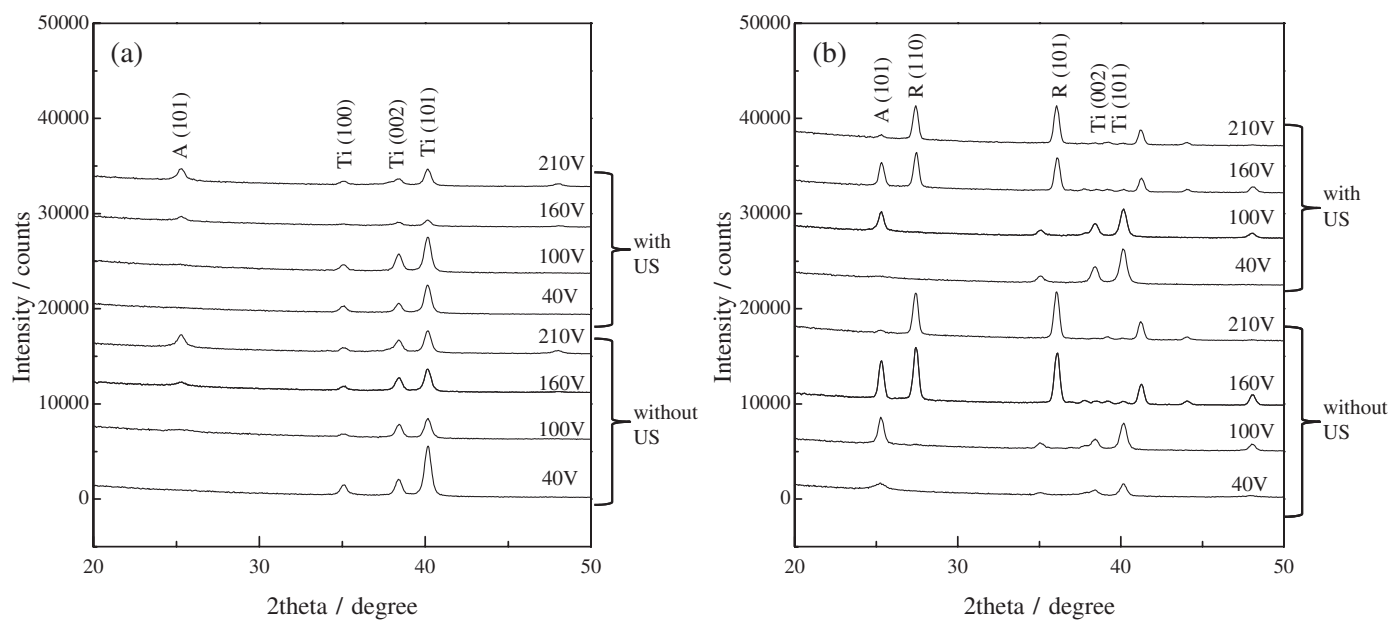

Fig. 3 XRD profiles of the as-anodized oxides prepared in an electrolyte of $0.02 \mathrm{M}$ (a), $0.79 \mathrm{M}$ (b) sulfuric acid concentration with and without ultrasonic irradiation (US).
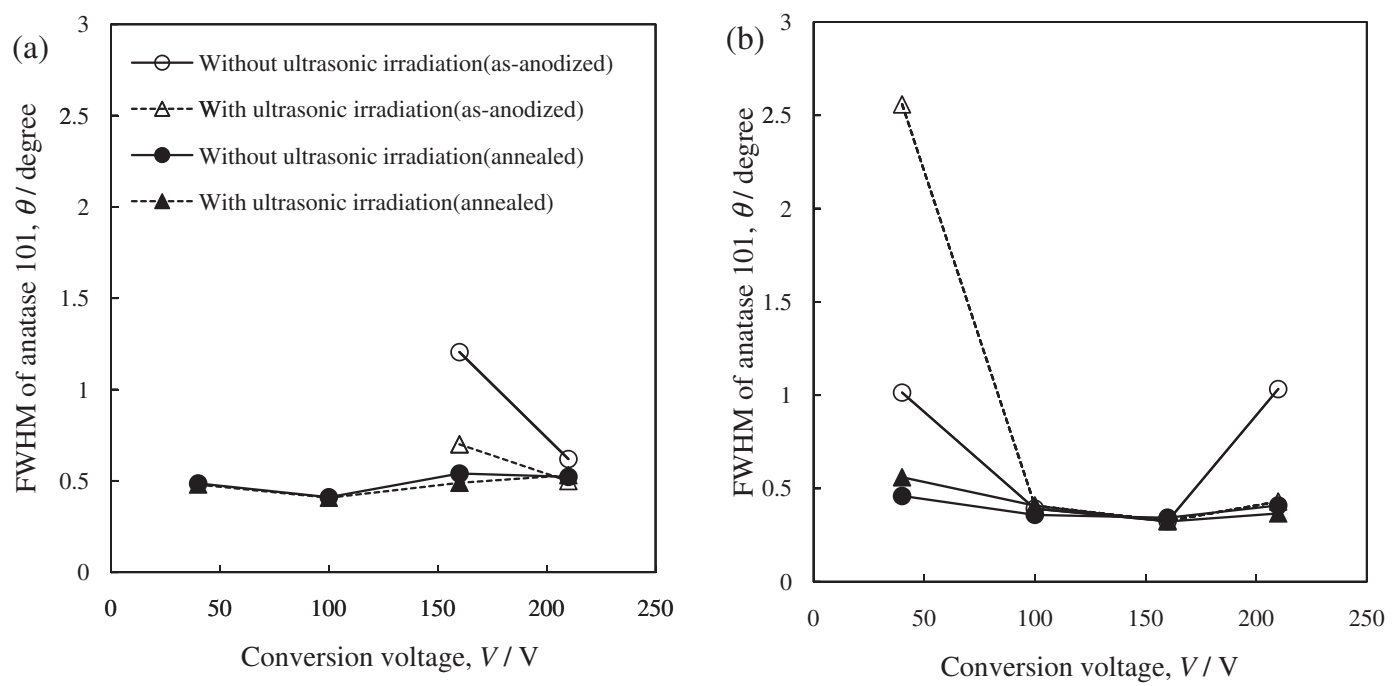

Fig. 4 FWHM of anatase (101) of the oxides anodized in $0.02 \mathrm{M}$ (a) and $0.79 \mathrm{M}$ (b) sulfuric acid against the conversion voltage.

an effect of ultrasonic irradiation on anodic oxide formation. It is speculated that ultrasonic irradiation promotes movements of constituents when the concentration of sulfuric acid is low, whereas it does not affect movements of constituents when the concentration of sulfuric acid is high. This means that the ultrasonic irradiation has the same effect on oxide formation with increasing sulfuric acid concentration or annealing. Lowering the concentration of the sulfuric acid or omission of high energy processes such as annealing is beneficial for practical processing due to reduction of the environmental burdens. Further studies are necessary to elucidate the mechanism of ultrasonic irradiation on oxide formation.

\subsection{Hydrophilicity}

Figure 5 shows the plots of the contact angles against the duration of UV light illumination. The contact angle of the oxides anodized in $0.02 \mathrm{M}$ sulfuric acid decreases with the duration of UV light illumination when the conversion voltage is $210 \mathrm{~V}$. The contact angle of the annealed oxides is lower than that of the as-anodized oxides as shown in (b).
Further, the contact angle of the as-anodized oxides is decreased with ultrasonic irradiation, regardless of the duration of UV light illumination as shown in (a). This is probably attributed to an improvement of the crystallinity because it promotes a generation of oxygen vacancy responsible for adsorption of hydroxyl groups under UV light illumination. ${ }^{17)}$ On the other hand, it is not recognized similar characteristics in the oxides when the sulfuric acid concentration is $0.79 \mathrm{M}$ (c), (d). This could be understood that the crystallinity is promoted with an increase of the sulfuric acid concentration in the electrolyte regardless of ultrasonic irradiation, thereby the effect of ultrasonic irradiation on hydrophilicity is not clear.

\subsection{Photocatalytic activities}

Figure 6 shows the plots of the degradation rates of MB against the conversion voltage. When the sulfuric acid concentration is $0.02 \mathrm{M}$, the annealed oxides show high activities as compared to the as-anodized oxides irrespective of the conversion voltage as shown in (a). The same characteristics are verified when the sulfuric acid concentration is 

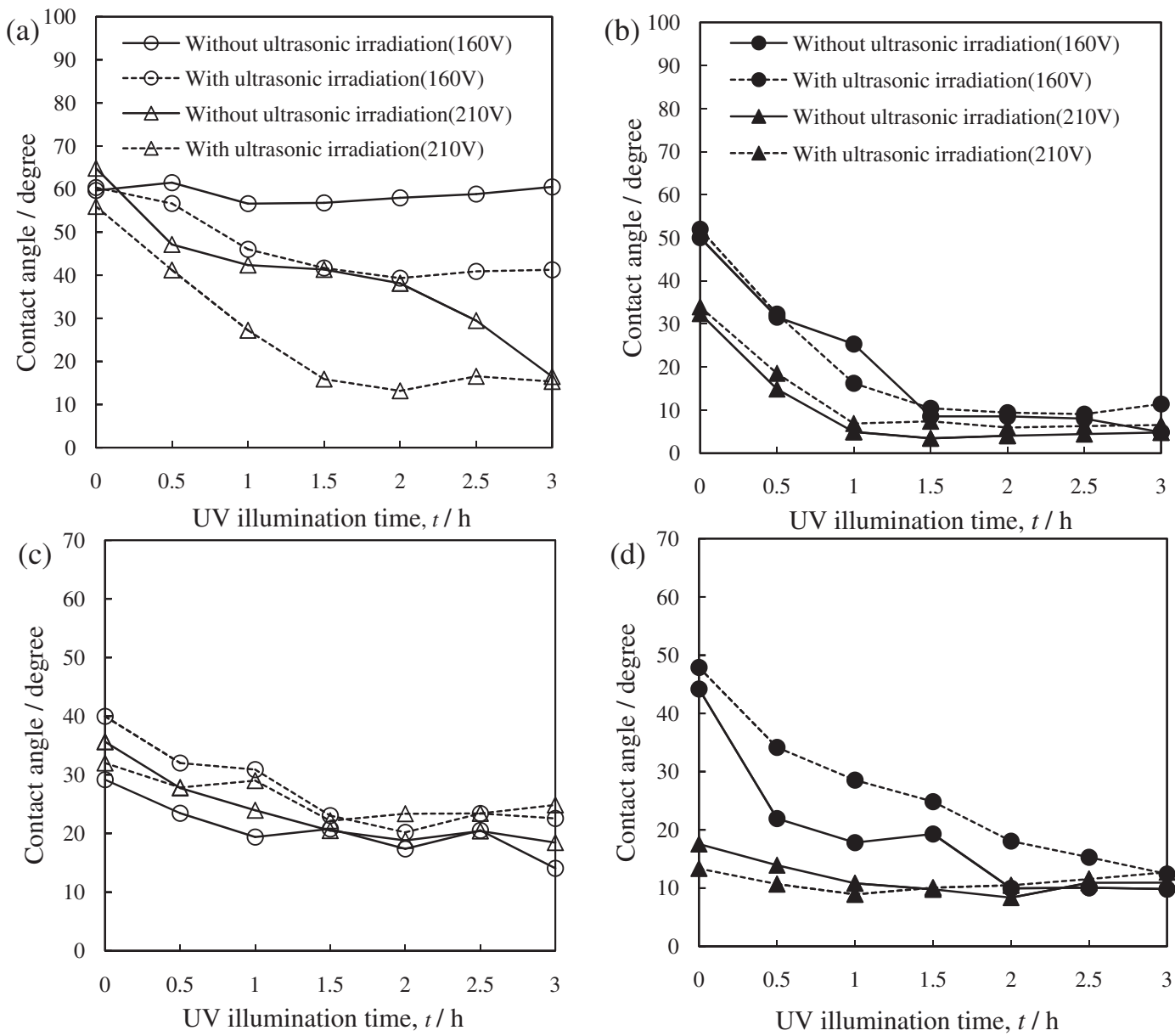

Fig. 5 Plots of the contact angles against the duration of UV light illumination for the oxides anodized in an electrolyte with sulfuric acid concentrations of $0.02 \mathrm{M}$; (a) as-anodized and (b) annealed, $0.79 \mathrm{M}$; (c) as-anodized and (d) annealed.
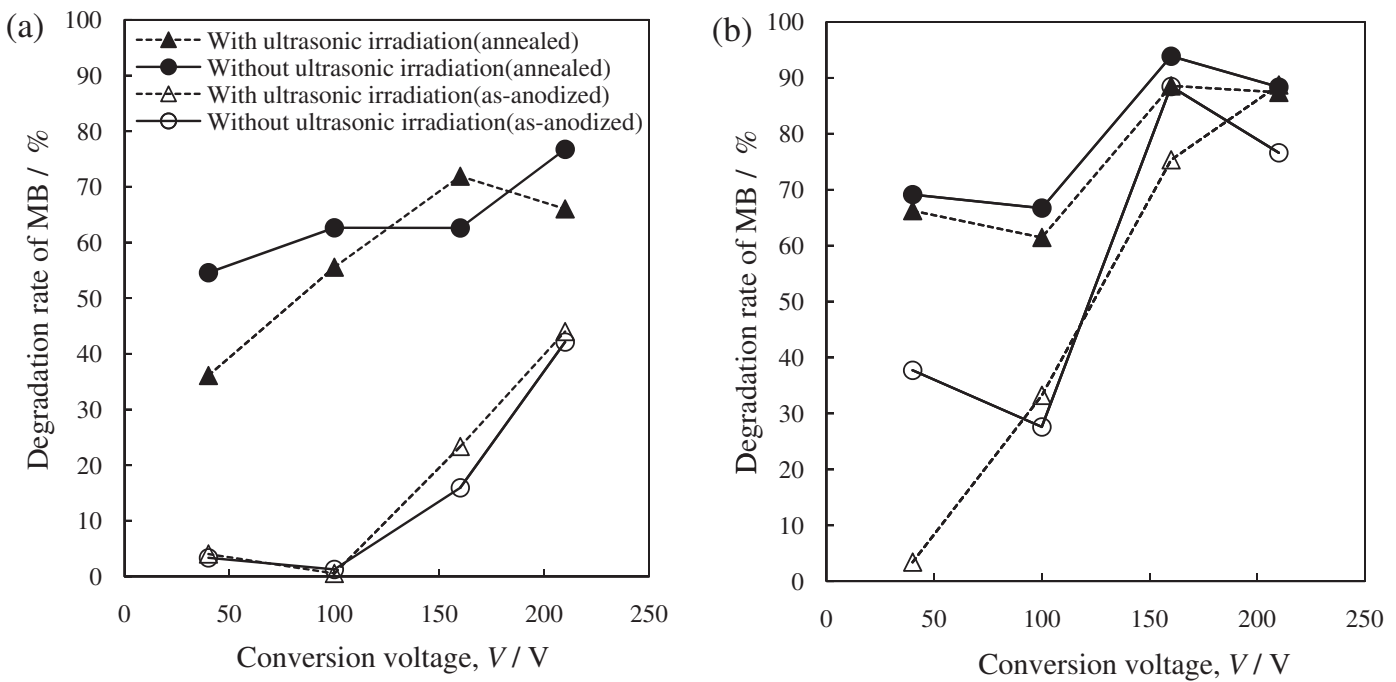

Fig. 6 Plots of the degradation rates of MB after UV illumination against the conversion voltage for the oxides anodized in an electrolyte with sulfuric acid concentrations of $0.02 \mathrm{M}$ (a) and $0.79 \mathrm{M}(\mathrm{b})$.

$0.79 \mathrm{M}$, and it is found that the photocatalytic activity of the anodic oxide prepared in an electrolyte of $0.79 \mathrm{M}$ sulfuric acid is higher than the corresponding of $0.02 \mathrm{M}$ irrespective of conversion voltage and annealing. In contrast, the photocatalytic activity was not improved by ultrasonic irradiation irrespective of the sulfuric acid concentration. This result is inconsistent with the fact that an improvement of the crystallinity leads to an enhancement of the photocatalytic activity due to the reduction of the recombination sites (e.g. point defects, dislocations etc.) of the photogenerated electron and hole. Consequently, it is considered that the photocatalytic activity in the present study could be affected 
by other factors such as the recombination rate of the electron and hole, the mobility of the electron and hole, and geometrical factors in the microstructure. Further studies have been under progress to elucidate it.

\section{Conclusions}

The photocatalytic characteristics of the anodic oxide focusing on the effect of ultrasonic irradiation were studied. Ultrasonic irradiation during anodic oxidation improves the crystallinity of the anodic oxides, and an application of ultrasonic irradiation has the same effect on improving crystallinity with annealing. The contact angles of the as-anodized oxides with ultrasonic irradiation were lower than those of the as-anodized oxides without ultrasonic irradiation. In contrast, the photocatalytic activity was not improved by ultrasonic irradiation regardless of annealing.

\section{REFERENCES}

1) A. Fujishima, T. N. Rao and D. A. Tryk: J. Photochem. Photobiol. C1 (2000) 1-21.

2) A. Gedanken: Ultrason. Sonochem. 11 (2004) 47-55.
3) L. H. Thompson and L. K. Doraiswamy: Ind. Eng. Chem. Res. 38 (1999) 1215-1249.

4) T. J. Masaon (Ed.): Advances in Sonochemistry vol. I, (JAI press, London, 1990) pp. 1-37.

5) W. B. McNamara III, Y. T. Didenko and K. S. Suslick: Nature 401 (1999) 772-775.

6) K. Makino, M. M. Mossoba and P. Riesz: J. Phys. Chem. 87 (1983) 1369-1377.

7) S. K. Doktycz and K. S. Suslick: Science 247 (1990) 1067-1069.

8) L. A. Crum: J. Acous. Soc. Am. 95 (1994) 559-562.

9) C. E. Banksa and R. G. Compton: Electroanal. 15 (2003) 329-346.

10) R. G. Compton, J. C. Eklund, S. D. Page, T. J. Mason and D. J. Walton: J. Appl. Electrochem. 26 (1996) 775-784.

11) S. K. Mohapatra, K. S. Raja, M. Misra, V. K. Mahajan and M. Ahmadian: Electraochim. Acta 53 (2007) 590-597.

12) S. K. Mohapatra, M. Misra, V. K. Mahajan and K. S. Raja: J. Catal. 246 (2007) 362-369.

13) N. Masahashi, S. Semboshi, N. Ohtsu and M. Oku: Thin Solid Films 516 (2008) 7488-7496.

14) N. Masahashi, Y. Mizukoshi, S. Semboshi and N. Ohtsu: Chem. Lett. 37 (2008) 1126-1127.

15) N. Masahashi, Y. Mizukoshi, S. Semboshi and N. Ohtsu: Appl. Catal. B 90 (2009) 255-261.

16) Y. Mizukoshi, N. Ohtsu, S. Semboshi and N. Masahashi: Appl. Catal. B (2009) in press.

17) N. Sakai, A. Fujishima, T. Watanabe and K. Hashimoto: J. Phys. Chem. B 105 (2001) 3023-3026. 